\title{
The contractile vacuole complex of protists - New cues to function and biogenesis
}

\author{
Helmut Plattner \\ Department of Biology, University of Konstanz, Konstanz, Germany
}

\section{Abstract}

The contractile vacuole complex (CVC) of freshwater protists sequesters the excess of water and ions $\left(\mathrm{Ca}^{2+}\right)$ for exocytosis cycles at the pore. Sequestration is based on a chemiosmotic proton gradient produced by a $\mathrm{V}$-type $\mathrm{H}^{+}$-ATPase. So far, many pieces of information available have not been combined to a comprehensive view on CVC biogenesis and function. One main function now appears as follows. $\mathrm{Ca}^{2+}$-release channels, type inositol 1,4,5-trisphosphate receptors $\left(\mathrm{InsP}_{3} \mathrm{R}\right)$, may serve for fine-tuning of local cytosolic $\mathrm{Ca}^{2+}$ concentration and mediate numerous membrane-to-membrane interactions within the tubular spongiome meshwork. Such activity is suggested by the occurrence of organelle-specific soluble $\mathrm{N}$-ethylmaleimide sensitive factor attachment protein receptor (SNARE) and Ras-related in brain (Rab) proteins, which may regulate functional requirements. For tubulation, F-Bin amphiphysin Rvs (F-BAR) proteins are available. In addition, there is indirect evidence for the occurrence of $\mathrm{H}^{+} / \mathrm{Ca}^{2+}$ exchangers (to sequester $\mathrm{Ca}^{2+}$ ) and mechanosensitive $\mathrm{Ca}^{2+}$-channels (for signaling the filling sate). The periodic activity of the CVC may be regulated by the mechanosensitive $\mathrm{Ca}^{2+}$ channels. Such channels are known to colocalize with and to be functionally supported by stomatins, which were recently detected in the CVC. A Kif18-related kinesin motor protein might control the length of radial arms. Two additional Ins $_{3}$-related channels and several SNAREs are associated with the pore. De novo organelle biogenesis occurs under epigenetic control during mitotic activity and may involve the assembly of $\gamma$-tubulin, centrin, calmodulin and a never in mitosis A-type (NIMA) kinase components also engaged in mitotic processes.
\end{abstract}

\section{Keywords}

Biogenesis, calcium, ciliate, contractile vacuole, Dictyostelium, osmoregulation, Paramecium, protozoa, Tetrahymena

\section{Introduction}

Many fresh water organisms possess a contractile vacuole complex (CVC) to counteract the permeation of water and ions, mainly $\mathrm{Ca}^{2+}$, by periodic expulsion of fluid (Allen \& Naitoh, 2002). The CVC consists of the contractile vacuole (CV) with radial arms and a spongiome attached; the latter is a widely branched tubular membrane system involved in fluid collection and extrusion. The $\mathrm{CV}$, the most impressive part of the CVC, is attached to the cell membrane at a specific site, called the pore. At the pore, the CV undergoes kiss-and-run type cycles of exo-/endocytosis. Radial arms, also called collecting canals, collect the fluid for transfer via ampullae to the CV. All ampullae display a transiently swollen or collapsed appearance during each activity cycle. See Figure 1.

The structure of the CVC varies depending on the species analyzed. However, CVCs from different systems share important basic aspects. Unfortunately, much information is buried as minor remarks in reports unrelated to the CVC. Therefore, it now appears reasonable to summarize and

Address for correspondence: Helmut Plattner, Department of Biology, University of Konstanz, P.O. Box M625, 78457 Konstanz, Germany. Tel: 00497531 2228. Fax: 00497531 2245. E mail: helmut.plattner@uni konstanz.de connect the manyfold new data. We may, thus, obtain an advanced understanding of basic principles of function and biogenesis of this complex, dynamic organelle. Most importantly, on this basis, many aspects will be amenable to experimental scrutiny in the future.

\section{Basic aspects of structure, function and organelle biogenesis}

In ciliates, microtubules emerge at the pore, surround the $\mathrm{CV}$ and extend over ampullae out to the tips of radial arms (Allen, 2000). In Dictyostelium, smaller vacuoles fuse to form the CV, called the bladder (Heuser et al., 1993). As CV, ampullae and radial arms collapse during contraction, their membrane is transformed into tubules (Gerisch et al., 2002; Heuser, 2006; Tominaga et al., 1998b). They thus form a membrane reservoir, which largely smooths out again during swelling. This reversible change, planar $\leftrightarrow$ tubular, is required because biomembranes cannot expand or shrink to any significant extent (Andersen \& Koeppe, 2007). In Paramecium, ampullae are known to be disconnected from the $\mathrm{CV}$ before the $\mathrm{CV}$ contracts (Tominaga et al., 1998a).

In more distant regions, radial arms are flanked by a stationary meshwork of tubular membrane extensions, the smooth spongiome (Figure 2). Close to the CV, this tubular network is variable in form and time, depending on the 
activity cycle. In the more distal parts of the radial arms, the smooth spongiome is continuous with the decorated spongiome, which harbors the proton pumps (Fok et al., 1993; Heuser et al., 1993; Nolta et al., 1993). The $\mathrm{H}^{+}$-ATPase holoenzyme is composed of a membrane-integrated base piece, V0, and a reversibly attached catalytic part, V1, both being composed of several subunits. V1 heads are seen in the electron microscope (EM) as pegs on the peripheral part of the spongiome. Because of its appearance, this part is called the decorated spongiome, in contrast to the smooth spongiome in the proximal part. There is agreement that this pump is the driving force for the sequestration of water and ions.

(a)

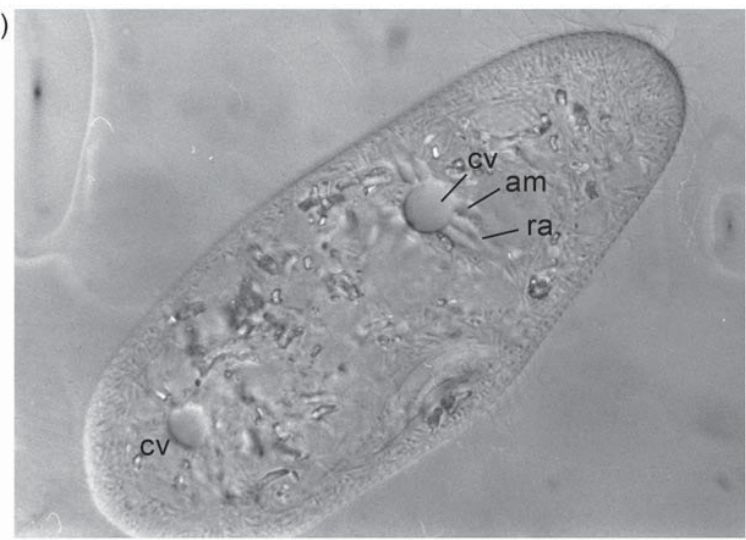

(b)

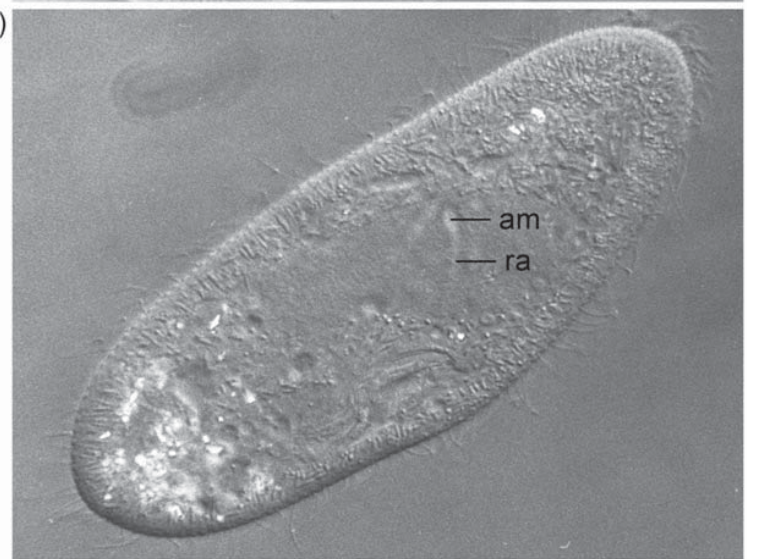

Figure 1. Light micrographs showing different functional stages of the CVC in P. tetraurelia. (a) shows two contractile vacuoles (cv), the one on top is nearly at the end of diastole; its radial arms (ra) are still attached to the vacuole via ampullae (am). (b) Presents a contractile vacuole in the stage of systole, when the ampullae are disconnected from the vacuole, which is no more visible. Magnification $600 \times$.
Its activity is based on a $\mathrm{H}^{+}$gradient created by the $\mathrm{V}$-type $\mathrm{H}^{+}$-ATPase, thus energizing the CVC membranes (Grønlien et al., 2002).

Some of the subsequent discussion is hypothetical, although the functions envisaged for the different CVC components is supported by well-established functions of individual metazoan proteins. For instance, the maintenance of osmotic and ionic balance requires the occurrence of both, aquaporin and $\mathrm{a} \mathrm{H}^{+} / \mathrm{Ca}^{2+}$ exchanger; however, no cation exchanger has been identified as yet in the CVC of any species. As often, database mining is one step but definitely not sufficient for localization to the CVC since such exchangers can occur at several different sites in a cell. Moreover, the occurrence of proteins engaged in vesicle trafficking deep inside the smooth spongiome needs an explanation as no overt vesicle trafficking can be recognized and since the dense packing of spongiome tubules would hinder vesicle delivery inside this structure. In this part of the CVC, the formation of membrane tubules is another challenging problem and even more their branching and debranching. Another important question: What is the significance of the occurrence of $\mathrm{Ca}^{2+}$ signals and of different types of $\mathrm{Ca}^{2+}$-release channels (CRC) within the organelle? Not even the most evident function of the $\mathrm{CV}$, i.e. periodic swelling (diastole) and contraction (systole), has been sufficiently explained up to now. For example, the occurrence of mechanosensors is very likely for reasons to be discussed, but this has not yet been analyzed and even considered unlikely. Some of the aspects subsequently discussed are extrapolated from well-established mechanisms known from other, mostly metazoan cells and organelles. In summary, this review is meant to provide a baseline for future research.

It is important to anticipate that membrane-to-membrane interactions from recognition and tethering to fusion involves mainly three types of proteins. These encompass the exocyst (Heider \& Munson, 2012), Rab-type GTPases (small GTP-binding proteins [Stenmark, 2009]), soluble NSF [N-ethylmaleimide sensitive factor] attachment protein receptor (SNARE) proteins (Jahn \& Scheller, 2006) and $\mathrm{Ca}^{2+}$ sensor proteins (Pang \& Südhof, 2010). Considering the participation of a vesicle and of a target membrane, SNAREs are subdivided into v-SNAREs and t-SNAREs or R- and Q-SNAREs according to the aminoacid in the central layer of the SNARE domain (Jahn \& Scheller, 2006). All these proteins are mandatory for vesicle-to-membrane (target) interaction. Their distribution is indicative of unexpected
Figure 2. Electron micrograph of the spon giome, including the smooth spongiome (ss) around a radial arm (ra) and the more peripheral decorated spongiome (ds). Magnification $15000 \times$.

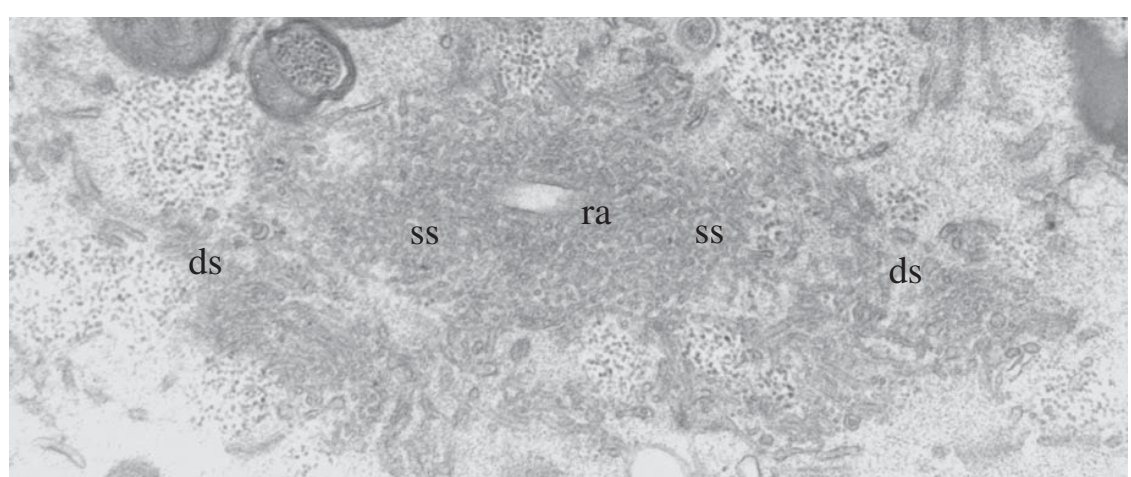


dynamics, from the pore down to the smooth spongiome. Recently, new insight into CVC function has been obtained by the discovery of Rab-type GTPases in Tetrahymena (Bright et al., 2010), of exocyst components in Chlamydomonas (Komsic-Buchmann et al., 2012) and of multiple SNARE proteins in Paramecium (Kissmehl et al., 2007; Schilde et al., 2006, 2008, 2010; Schönemann et al., 2013).

Considering the expulsion of $\mathrm{Ca}^{2+}$ (and other ions) by the CV of Paramecium (Stock et al., 2002), it was surprising to find that the CVC contains CRCs by which it can release some $\mathrm{Ca}^{2+}$ back into the cytosol (Ladenburger et al., 2006; Ladenburger \& Plattner, 2011). Moreover, the recent discovery of the scaffolding protein, stomatin, also in the CVC of Paramecium (Reuter et al., 2013), suggests the occurrence of mechanosensitive $\mathrm{Ca}^{2+}$ channels in the CVC. We conclude this from the following three facts: (i) stomatin and mechanosensitive $\mathrm{Ca}^{2+}$ channels are known to colocalize, (ii) also to be functionally interdependent in all other systems analyzed (Huang et al., 1995) and (iii) in Paramecium, osmoregulation is disturbed after stomatin silencing (Reuter et al., 2013). We argue for the existence of such channels in the CVC in contrast to previous literature (Tani et al., 2001).

A battery of proteins has been localized to the pore, not only in Paramecium but also in Tetrahymena. Among them are SNAREs, CRCs, kinases and cytoskeletal elements. According to our view and hints from the literature, they seem to cooperate, together with other components, in the enigmatic regulation of de novo-biogenesis of the CVC. In Paramecium, new small CVCs are formed anterior to each of the old ones at the onset of cytokinesis (Allen et al., 1990), and only rarely laterally to the old organelles (Klotz et al., 2003). In ciliates, this process is under epigenetic control (Frankel, 2000; Klotz et al., 2003) and rather poorly understood. However, from work with different systems unexpected cues emerge also for this aspect. A question concerning the far ends of the organelle: Which mechanism trims radial arms in the CVC of ciliates to equal size?

A literature search shows that altogether almost one hundred proteins are currently known from the CVC of different species, based on molecular biology combined with localization studies. Considering basic similarities in structure and function of the CVC in different species, this may suffice to address old questions concerning function and biogenesis and to predict new paradigms and hypotheses for future research. For a schematic summary, see Figure 3.

\section{Proteins involved in vesicle trafficking}

Proteins engaged in vesicle trafficking may be important not only for de novo organelle biogenesis but also for invisibly ongoing turnover (removal of old and delivery of new proteins). One may call this "overt" and "hidden" biogenesis, respectively, since the de novo formation of the organelle can be followed in the microscope, in contrast to the replacement of molecular components by steadily ongoing delivery of vesicles of submicroscopic size. Moreover, some proteins may serve for restructuring of membranes within the organelle in the course of its normal activity, as will be discussed subsequently. Therefore, membrane fusion events occurring in the CVC may also be classified as "overt" and "hidden", respectively. It is advisable to make this distinction in order to explain the occurrence of proteins, such as SNAREs, in regions where vesicle trafficking and fusion cannot be seen with the usual methods, although fusion/fission of membrane tubules may take place in a reversible manner, for instance within the smooth spongiome (below). This may also help to appreciate the CVC as a highly dynamic organelle far beyond its fascinating pulsations visible in the light microscope.

\section{SNAREs, Rabs, exocyst - components for membrane fusion and fission}

All three protein types are required for vesicle docking at a partner membrane and fusion. The relevance of the exocyst complex, required for tethering a vesicle to another membrane, has been established for CVC function in Chlamydomonas (Komsic-Buchmann, 2012). Rab-type proteins have been localized to the CVC in Tetrahymena thermophila and also occur in Paramecium tetraurelia (Pt) (Bright et al., 2010). Among t-/Q-SNAREs syntaxin (Syx) type PtSyx2, PtSyx14 and PtSyx15 (Kissmehl et al., 2007) and among v-/R- SNAREs synaptobrevin (Syb) type PtSyb2, PtSyb6 and PtSyb9 have been localized to the CVC of Paramecium (Schilde et al., 2006, 2010), together with a rather ubiquitous t-/Qa,b-SNARE, $P$ t-soluble NSF attachment protein-25-like (SNAP-25) protein (Schilde et al., 2008).

The pore and the CV/ampullae transition contain the SNARE-specific chaperone NSF in high density. However, NSF also occurs in other regions of the CVC except the decorated spongiome (Kissmehl et al., 2002). First of all, the presence of SNAREs indicates that fusion processes in the organelle cannot be merely based on physical forces, as previously suggested (Sugino et al., 2005), particularly since silencing of CVC-resident SNAREs greatly affects organelle function (Schönemann et al., 2013). "'Overt' membrane fusion is visible at the pore and can be detected also at CV/ampullae connections by membrane capacitance measurements (Sugino et al., 2005). In contrast, "hidden" fusion events occurring within the spongiome are not amenable to any of the analytical methods available. Only the occurrence of "hidden fusion" can explain the occurrence of both v- and t-SNAREs deep inside the smooth spongiome (Kissmehl et al., 2007; Schilde et al., 2006, 2010) where no vesicle transport is known to occur.

Furthermore, the visualization of the SNARE-specific chaperone, NSF, under appropriate conditions (Kissmehl et al., 2002) and of a battery of SNARE proteins (Kissmehl et al., 2007; Schilde et al., 2006, 2008, 2010) over most parts of the CVC in Paramecium indicates permanently ongoing membrane vesicle trafficking and/or restructuring activity. Remarkably, no trafficking vesicles have been identified, but steady state biogenesis may be a "hidden", event. It can serve for molecular turnover. Only in rare cases, small, electron-dense vesicles, possibly stages of autophagy, are recognized within the smooth spongiome (unpubl. observ.).

Nevertheless, vesicle delivery whatever the accessible sites may be is required for maintaining the ultrastructure of the smooth spongiome. This becomes evident from the 
obliteration of the smooth spongiome after silencing of NSF (Schilde et al., 2010) or of different organelle-resident SNAREs (Schönemann et al., 2013). Considering the usual silencing times of $\geq 65 \mathrm{~h}$, obliteration of the smooth spongiome can equally well be due to disturbed de novo biogenesis. The dense packing of the membrane meshwork may preclude vesicles from penetrating into the smooth spongiome. However, since SNAREs are also localized to the radial arms and the $\mathrm{CV}$, they may serve not only for vesicle docking and fusion in these areas but also for restructuring during fusion processes that accompany reversible planar $\leftrightarrow$ tubular transitions of the membranes. There are good reasons for this assumption as outlined below.

Hypothetically, SNAREs may also serve for membrane fusion events in the course of local restructuring of the smooth spongiome. This would enable the de-/re-coupling of parts of the spongiome, with a useful feedback to the water/ ion sequestration activity, depending on the physiological requirements. In fact, the fusion capacity of fragments of isolated CVCs has been documented in vitro (Sugino et al., 2005). Due to their high free energy, tubular protrusions are expected to be very liable to fusion, as shown by old (Blumenthal, 1987; Ohki, 1988) and very recent analyses (Shilagardi et al., 2013). Reversible restructuring would also require membrane fission events, not only for the transition from branched tubular elements to planar membranes (along the radial arms/smooth spongiome continuum) but also for disconnecting tubules within the continuum of the smooth spongiome. Since no dynamin has been detected as yet outside the pore (Tetrahymena [Elde et al., 2005]), one may postulate the existence of dynamin-like proteins for this activity, as known from some organellar membranes in metazoan cells (Ren et al., 2006; Yoon et al., 1998).

\section{F-Bin-amphiphysin-Rvs proteins}

Tubular extensions formed during systole can provide a membrane reservoir when the organelle swells during diastole. An interplay between membrane collapse during systole (with tubularization) and swelling during diastole (with rounding up) appears mandatory considering the fact that biomembranes are not stretchable (Andersen \& Koeppe, 2007). Prerequisite for tubularization is a mechanism for establishing this conformation. As known from metazoan cells and from in vitro studies, tubularization of intracellular membranes can be achieved by several proteins, including F-Bin amphiphysin Rvs (F-BAR) proteins (Mim \& Unger, 2012; Shen et al., 2012). These have been shown to occur in the CVC of Dictyostelium (Heath \& Insall, 2008), although no functional implications have been derived from this finding.

\section{$\mathrm{H}^{+}$-ATPase, aquaporin and cation exchangers}

The documentation of the $\mathrm{H}^{+}$-ATPase/pump in Paramecium (Fok et al., 1993) and Dictyostelium (Fok et al., 1993; Heuser et al., 1993; Nolta et al., 1993) shows localization exclusively in the decorated spongiome, which forms a continuum with the proximal smooth spongiome. The composition of subunits differs, in part, from that in other organelles (Wassmer et al., 2006, 2009). Inhibition by concanamycin B in Paramecium disturbs CVC activity (Grønlien et al., 2002) and indirectly
$\mathrm{Ca}^{2+}$ homeostasis (Plattner et al., 2012). As discussed above, the primary active organellar $\mathrm{H}^{+}$-ATPase/pump enables the chemiosmotic sequestration of excess water and ions, among them $\mathrm{Ca}^{2+}$.

Aquaporin has been identified in the CVC of Amoeba proteus (Nishihara et al., 2008) and, by proteomics, of Trypanosoma cruzi (Montalvetti et al., 2004). Exchangers, e.g. for $\mathrm{Ca}^{2+} / \mathrm{H}^{+}$, may be postulated because $\mathrm{Ca}^{2+}$ is secreted by the CVC of Paramecium (Stock et al., 2002) in the alleged absence of a $\mathrm{Ca}^{2+}$-pump in the CVC. In Paramecium, this has been ascertained for the sarcoplasmic/endoplasmic reticulum $\mathrm{Ca}^{2+}$-ATPase type pump, which clearly is absent from the CVC (Hauser et al., 2000). However, the absence of a plasma membrane $\mathrm{Ca}^{2+}$-ATPase (PMCA-type pump) may be disputable because the antibodies previously used may not have discovered all PMCA forms at all sites of the Paramecium cell (Yano et al., 2013). Indeed, PMCA has been localized to the CVC of Dictyostelium (Marchesini et al., 2002).

\section{Conclusions on the possible function of SNAREs in the CVC}

As mentioned, there is no overt vesicle trafficking recognizable in microscopic studies outside the pore and the CV/radial arms junctions. However, SNAREs and Rab proteins are scattered over the CVC in Paramecium and Tetrahymena, respectively. It is reasonable to hypothesize that these, together with exocyst components, can play a role during internal restructuring by reversible dis-/reconnection of spongiome components. According to this novel hypothesis, a dynamic adjustment to the functional requirements could thus be achieved. Apart from this hypothetical function, these proteins may also serve for the delivery of some of the organelle-resident proteins, i.e. renewal of components. Only the decorated spongiome may require indirect pathways or non-vesicular transport of $\mathrm{H}^{+}$-ATPase components.

\section{Inositol 1,4,5-trisphosphate receptor/ryanodine receptor-type CRCs and mechanosensitive $\mathrm{Ca}^{2+}$-channels}

Six subfamilies of CRCs, comparable in important features to inositol 1,4,5-trisphosphate receptor $\left(\mathrm{InsP}_{3} \mathrm{R}\right)$ and ryanodinereceptors (RyR), have recently been identified in $P$. tetraurelia cells, three of them in the CVC (Ladenburger \& Plattner, 2011). These CRCs share several properties with their homologs in mammalian cells, but the Paramecium proteins have variable inositol 1,4,5-trisphosphate ( $\mathrm{InsP}_{3}$ )-binding domains (BD). Subfamily II CRC members, PtCRC-II, possess an experimentally verified $\mathrm{InsP}_{3}$-BD and are localized to the smooth spongiome, the radial arms and the $\mathrm{CV}$ membrane (Ladenburger et al., 2006). Activation experiments with caged $\mathrm{InsP}_{3}$ and gene silencing experiments strongly argue in favor of $\mathrm{Ca}^{2+}$ signaling within the CVC; in fact, spontaneous signals are frequently observed, thus indicating constitutive channel activity. Among PtCRC-V members, only subtype $P t \mathrm{CRC}-\mathrm{V}-4$ has a predicted $\mathrm{InsP}_{3}-\mathrm{BD}$ and is the only one found rather selectively in the $\mathrm{CV}$ (Ladenburger \& Plattner, 2011). An additional CRC, type PtCRC-VI, is devoid of an $\mathrm{InP}_{3}$-BD and localizes rather selectively to the pore (Ladenburger \& Plattner, 2011). 
Most recently, the scaffolding protein stomatin has been identified in P. tetraurelia (Reuter et al., 2013); stomatin types Sto1p and Sto4p have been seen in association with the CVC. For reasons discussed above, the presence of Stomatin in different parts of the organelle (smooth spongiome, the radial canals, the $\mathrm{CV}$ and the pore region) suggests the presence of mechanosensitive channels, particularly since their colocalization is obligatory in the systems analyzed so far (Lapatsina et al., 2012).

In Chlamydomonas, the CVC contains another mechanosensitive channel, type-transient receptor potential (TRP) channel TRP5 (Fujiu et al., 2011). The Paramecium genomic database also contains TRP-type channels (Plattner et al., 2012) and in addition a homolog of mammalian mechanosensitive channels, type Piezo (Coste et al., 2010). Their molecular identification, characterization as CRCs and localization, though important, remains to be established.

\section{Rationale of mechanosensation}

In Paramecium, different types of CRCs related to $\mathrm{InsP}_{3} \mathrm{R} /$ RyRs occur in the CVC, from the pore to the smooth spongiome. Depending on the system, there is direct or indirect evidence of different mechanosensitive channels in the CVC. Tensions measured in the CV of Paramecium (Tani et al., 2001) are compatible with the general pressure sensitivity of mechanosensitive channels (Kung et al., 2010). The cyclic activation of such channels at maximal organelle pressure may yield a $\mathrm{Ca}^{2+}$ signal for inducing membrane fusion, first at the ampullae/CV junction before $\mathrm{CV}$ systole and then, during $\mathrm{CV}$ systole, at the pore.

\section{Cytoskeletal components}

Microtubules with acetylated $\alpha$-tubulin, as they have been demonstrated to occur in the CVC of Tetrahymena (Gaertig et al., 1995), could contribute to the stretching of the radial arms with the smooth spongiome attached because the microtubules course over the CV membrane surface to the tips of the radial arms. Thus, they are probably responsible for the star-shape of the CVC in ciliates (Figure 1). From experience with metazoan cells, one may expect an antagonistic effect of plus- and minus-end directed motor proteins so that the radial arms are pulled in both directions along microtubules. Note that the minus end is at the pore where $\gamma$ tubulin is found in Tetrahymena (Shang et al., 2002) and in Paramecium (Klotz et al., 2003) and from where microtubules grow during de novo organelle biogenesis (see below). Candidates for motor proteins would be kinesin and dynein, as these pull membranes, e.g. the endoplasmic reticulum, along microtubules in opposite directions (Palmer et al., 2005). Since, however, no kinesins are currently known from the CVC of any species, actin and myosin would be an alternative for producing such tension. In reality, Myosin $\mathrm{J}$ in Dictyostelium has been functionally localized only to the pore (Jung et al., 2009), and no actin has ever been reported for any CVC. However, some actin isoforms in protozoa possess no signatures for binding those drugs that are commonly used for fluorescence localization, at least in Paramecium (Sehring et al., 2007). Also note that short actin filaments may not be easily recognized in the EM. In addition, some other linker proteins may be associated with calmodulin, which accompanies microtubules along radial arms in Paramecium (Fok et al., 2008).

Strikingly, radial arms are of nearly identical length which, therefore, must be strictly controlled. A possible explanation would be steady-state equilibrium, with identical kinetics of spontaneous de-/re-polymerization, in each of the arms. Alternatively, a kif18-related microtubule-depolymerizing kinesin species might trim microtubules to equal length, but this is known so far only from metazoan cells (Mayr et al., 2007; Niwa et al., 2012). As discussed below, microtubules are of paramount importance for the de novo biogenesis of the CVC.

\section{Hypothesis}

The regular shape of the CVC in ciliates and its attachment at the cell membrane involves regularly arranged microtubules. Links between microtubules and closely attached membranes are visible. Possibly motor proteins, including a kif18-like kinesin, would expand the organelle membranes and control the length of radial arms by moving cargos and controlling polymerization/depolymerization cycles.

\section{Pore components: $\mathrm{Ca}^{2+}$-binding proteins, tubulins, myosin and a kinase}

The pore is a restricted area at the cell surface where, instead of an alveolar sac (cortical $\mathrm{Ca}^{2+}$ store), a $\mathrm{CV}$ is attached in a broad contact area (Figure 3). It is important not only for the activity of the mature organelle but also for its de novo biogenesis.

Periodic kiss-and-run exo-/endocytosis cycles at the pore imply a pivotal role of Rab-type GTPases of SNAREs and of SNARE-associated proteins. In the pore region, Rab8 has been detected in Dictyostelium (Essid et al., 2012) as well as several SNARE species in Paramecium (as specified below), and a mammalian uncoordinated-18 (Munc-18) homolog a SNARE-associated protein in Dictyostelium (Sriskanthadevan et al., 2009; Essid et al., 2012). By implication, exocyst proteins, also found in the CVC of Dictyostelium (KomsicBuchmann et al., 2012), can be assumed to precede any SNARE activity. In Paramecium, the pore region contains $P t$ Syb2 and $P t$ Syb9 as well as $P t$ Syx 2 and $P t$ Syx 15 (Kissmehl et al., 2007; Schilde et al., 2006; Schönemann et al., 2013). Under appropriate conditions, NSF can be retained and visualized at the pore (Kissmehl et al., 2002).

Occurrence of dynamin and of adaptor protein AP-2 at the pore of Tetrahymena (Elde et al., 2005) implicates endocytotic membrane re-uptake via clathrin-coated pits. Since the occurrence of clathrin-coated pits has been visualized only in Dictyostelium (Heuser, 2006) and not in ciliates, this aspect of membrane recycling clearly remains to be settled for the different systems.

A number of proteins dealing with $\mathrm{Ca}^{2+}$ signaling are also assembled at the pore. In Tetrahymena, $\mathrm{Ca}^{2+}$-binding proteins include calmodulin (Numata \& Gonda, 2001) and centrin, Cen4 (Stemm-Wolf et al., 2005). In Paramecium, this is complemented by CRCs whose overall sequence is related to $\mathrm{InsP}_{3} \mathrm{R}$ and RyRs, including $\mathrm{PtCRC}$-VI-2 and $\mathrm{PtCRC}-\mathrm{VI}-3$ 
(Ladenburger \& Plattner, 2011). However, none of them contains an $\mathrm{InsP}_{3}$-BD or a $\mathrm{RyR} / \mathrm{InsP}_{3} \mathrm{R}$ homology domain and, therefore, their functional role remains elusive. In addition, the scaffolding protein, stomatin, type Stolp and Sto $4 p$, occurs at the pore (Reuter et al., 2013). Since stomatin is known to position mechanosensitive $\mathrm{Ca}^{2+}$-channels in metazoa (Lapatsina et al., 2012), its occurrence in the CVC of Paramecium may indicate the presence of such channels at the pore, in addition to other parts of the CVC (see above). Their presence suggests the formation of periodic $\mathrm{Ca}^{2+}$ signals at the pore by activation via internal pressure at the end of diastole to induce exocytotic membrane fusion at the time of maximal CV expansion.

Cytoskeletal components detected at the CVC pore in Tetrahymena include $\gamma$-tubulin (Shang et al., 2002), acetylated $\alpha$-tubulin (Gaertig et al., 1995) and a never in mitosis A (NIMA) type protein kinase (Wloga et al., 2006). In mammalian neurons, this kinase serves for the organization of the microtubular apparatus (Chang et al., 2009). A type V myosin (MyoJ) is required for $\mathrm{CV}$ docking in Dictyostelium (Jung et al., 2009).

\section{Hypothesis}

In summary, the pore contains many components, which are important for CVC function, i.e. periodic kiss-and-run exo-/ endocytosis cycles. For membrane fusion, the pore is endowed with a set of SNAREs and probably also with Rab and exocyst proteins. Specific $\mathrm{Ca}^{2+}$-channels, probably also including mechanosensitive types, may provide the $\mathrm{Ca}^{2+}$ signal required for this activity, although this still remains to be documented unequivocally. It is also difficult to imagine whether fusion occurring at the pore is of the usual focal type (point fusion), since at the pore the cell membrane and the CV membrane are closely apposed to each other over an unusually large area (Allen, 2000). Although this would be compatible with membrane fusion over a large area, current concepts of membrane fusion all advocate for point fusion (Plattner, 2010). To settle this question, one may consider patch-clamp analysis an appropriate method, but the large size of the protozoan cells under consideration and their rigid surface would make such analyses difficult particularly with ciliates. Closing of the pore may or may not include exocytosis-coupled endocytosis. As mentioned, dynamin would be available for membrane retrieval; but the precise mode how the pore is closed remains to be established and may not be the same, for instance in Dictyostelium and in ciliates.

\section{Hypothesis to de novo organelle biogenesis}

Based on the arguments presented, Figure 3 summarizes a hypothetical sequence of events for the biogenesis of the CVC in Paramecium. Most aspects discussed in this study are debatable, particularly when paradigms are derived from higher eukaryotes, but they may set a baseline for scrutiny in the future.

\section{Site of de novo formation of CVCs in Paramecium and its molecular inventory}

In Paramecium, a new CVC emerges before cytokinesis anterior to each of the two old CVCs. New CVCs are formed at the endpoint of that radial arm that extends parallel to the cell axis (Allen et al., 1990). More rarely, new CVCs are seen to nucleate, in log-phase cells, at a short distance in lateral position to the old ones (Klotz et al., 2003). Therefore, the nucleation signal for the assembly of a primordial scaffold, whose components are not known, may somehow come from the inherited CVCs. In each case local $\gamma$-tubulin assembly is an early step and, therefore, probably important for the formation of a primordial nucleation site, i.e. for initiating de novo CVC biogenesis. However, a molecule serving as a nucleator that may ultimately determine this primary step is not known.

The sites where a pore region will be installed must be special insofar as the attachment of alveolar sacs, which otherwise cover the entire cell surface, has to be avoided to make a site available for the docking of the CVC. Generally, cortical components are profoundly restructured before cytokinesis (Iftode et al., 1989), i.e. at the time of CVCs formation. Therefore, different signals may have to cooperate to trigger local $\mathrm{CVC}$ biogenesis. One may speculate that some of the signals may be linked to cell division activity. Changes in the local concentration of $\mathrm{Ca}^{2+}$ could play a role, possibly in conjunction with calmodulin and centrin. In fact, calmodulin and centrin, isoform Cen4, occur at the pore in Tetrahymena (Numata \& Gonda, 2001) and in Paramecium (Stemm-Wolf et al., 2005), together with $\gamma$-tubulin. Nevertheless, it may be mere coincidence that all these components are also found in metazoan centrioles (Dobbelaere et al., 2008; Hodges et al., 2010) and in functional counterparts, the basal bodies of ciliates (Gogendeau et al., 2008; Momayezi et al., 1986; Shang et al., 2002).

Globally, the first crucial aspect seems, thus, to be the nucleation of a primordial assembly site and positioning of the pore region. The second event will be the growth of a newly forming CVC, in conjunction with the outgrowth of the radial arms to defined length and the attachment of the membranes. Finally, the integration of the proton pumps makes the newly formed CVCs functional.

\section{Cell polarity and surface pattern in Paramecium}

A Paramecium cell displays considerable regularity of its surface pattern, and it also has an inherent vertical polarity, with microtubule organizing centers at the cell periphery (Plattner et al., 1982), rather than at a cytocenter near the nucleus. This resembles the situation in epithelial cells, in T-lymphocytes as well as at the cleavage furrow (Griffiths et al., 2010). Microtubules emanating in great number from the pore region to the tip of each radial arm have the same polar arrangement, with the minus-end at the pore. In some way, elements of the regular surface pattern could act as a scaffold for precise positioning of newly forming CVCs in ciliates. However, in Tetrahymena, disorganizing ciliary rows by mutation of the gene disA does not cause malpositioning of the CVC (Jerka-Dziadosz et al., 1995). This is in line with the fact that, upon spontaneous slippage of ciliary rows around the cell, a particular row can contribute to any part of the cellular pattern (Iftode et al., 1989). At this point, one can conclude that cell polarity, rather than the surface 


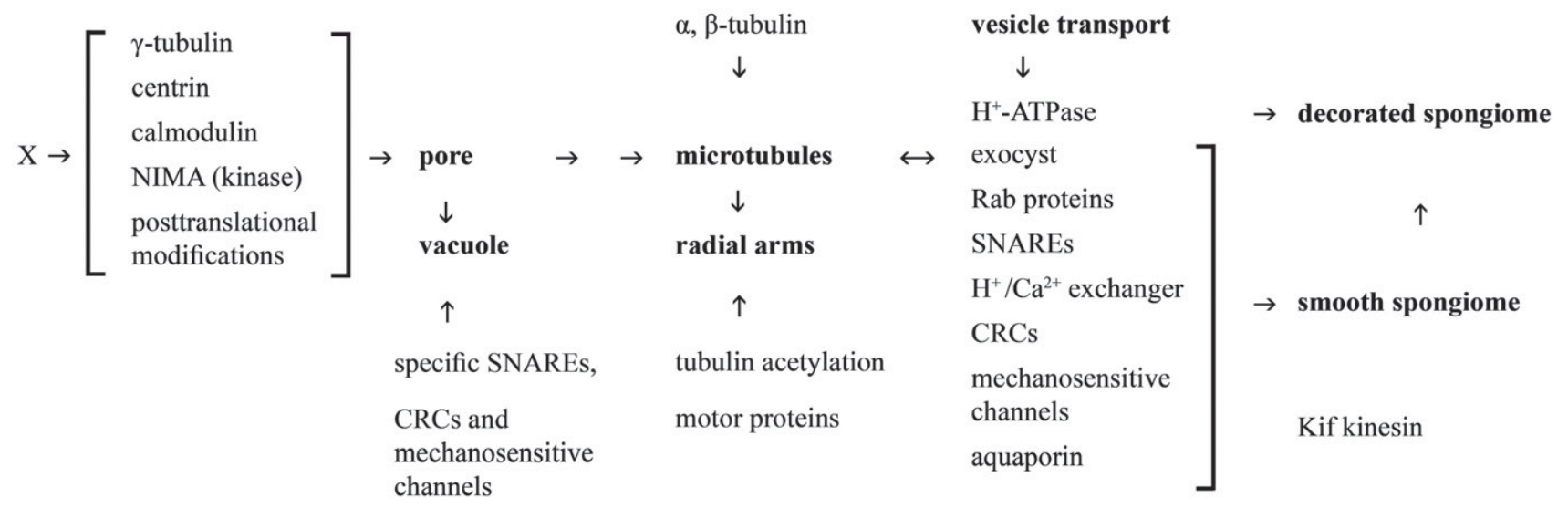

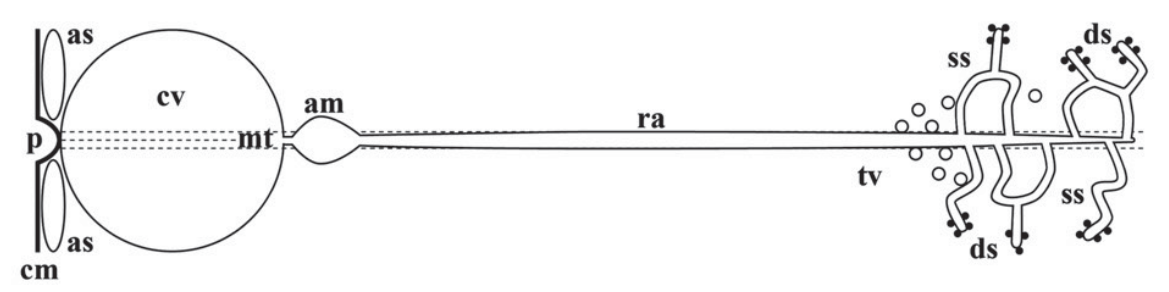

Figure 3. Schematic arrangement of proteins and their distribution in the CVC, based on data from different systems (see text). The arrows suggest possible molecular interactions (vertical arrows) and sequential events during de novo CVC biogenesis (horizontal arrows), respectively. Biogenesis starts at the cell membrane (cm) in a specific region devoid of alveolar sacs (as). A most early step of self assembly is designated " $\mathrm{X}$ " because its molecular nature is not yet known. This primary nucleation site allows for subsequent installation of the contractile vacuole complex, starting with the pore (p). Molecules indicated between brackets (including $\gamma$ tubulin) are assembled at the pore which then serves for the attachment of the contractile vacuole (cv) by delivery of vesicles with SNAREs and different ion channels. Polymerization of tubulin, including acetylated $\alpha$ tubulin and $\beta$ tubulin, is prerequisite for the formation of radial arms (ra), which in Paramecium are connected to the vacuole via ampullae (am). Radial arms receive membranes containing components relevant for vesicle trafficking (exocyst, Rab and SNARE proteins), ion exchangers (hypothetical) and different channels, thus forming the smooth spongiome (ss). The transport pathways for components of the multimeric $\mathrm{H}^{+}$ATPase may be different and are not yet known in detail. These molecules are heavily enriched in the decorated spongiome (ds), which is attached to the smooth spongiome, thus forming an anastomosing tubular network. The entire organelle is supported by microtubules (mt) whose length may be restricted by a Kif type kinesin, which hypothetically may trim radial arms to equal length.

pattern per se, will contribute to de novo biogenesis of the CVC in ciliates.

It is also difficult to understand why new CVCs are forming mostly anterior (Allen et al., 1990), but occasionally also lateral to the old organelles (Klotz et al. 2003). In the further discussion, the two modes are designated as "biogenesis type I' for anterior and "biogenesis type II" for lateral de novo formation of a CVC.

It remains to be seen whether specific morphogens and polarity genes come into play, as they are known from the development of cell polarity in metazoans. The functional domain, Hog, an ortholog to the metazoan morphogen Hedgehog occurs in some groups of protozoa that possess a CVC (Ingham et al., 2011). For Tetrahymena, five forms are listed in the Superfamily Backup server (supfam.cs.bris. ac.uk/), but neither their localization nor their function is known. Even less is known about the classical polarity gene families called $a P K C$ and Par, which are important in metazoans (Harris \& Peifer, 2005; Henrique \& Schweisguth, 2003; Joberty et al., 2000).

\section{Cytoskeletal elements}

Could tubulins play a role in the initiation and growth of a new CVC? As mentioned, the presence of centrin, calmodulin and $\gamma$-tubulin at the $\mathrm{CV}$ pore in ciliates strikingly recalls the organization of metazoan centrioles, the established microtubule organizing center for microtubule formation and center of vesicle trafficking. $\gamma$-tubulin, once installed for a newly forming pore, could serve as a nucleator for microtubules, which then elongate to support the formation of radial arms.

In biogenesis type I, the "partner" radial arm of the old CVC shrinks at the same time as the new CVC grows, as outlined by Allen et al. (1990). This raises several questions. First, is such a depolymerization compatible with the stability profile of acetylated $\alpha$-tubulin? This possibility has been demonstrated recently for mammalian cells (Kalebic et al., 2013), where it requires tubulin acetyltransferase $\alpha$ TAT1. However, in protozoa, this enzyme has not been looked for as yet. Second, could depolymerization of the microtubules of the old CVC arms be driven by Kif-type kinesin, which in metazoan cells drives microtubule depolymerization (Mayr et al., 2007; Niwa et al., 2012)? Again such kinesin subtypes have not yet been explored in cells possessing a CVC. Third, could an explorative microtubule, i.e. one from the old CVC, serve as a nucleator for a new $\mathrm{CVC}$ by inducing the formation of a new cell membrane-bound microtubule organizing center? This is indeed what takes place in the search-capture function in a variety of metazoan cells (Desai \& Mitchison, 1997) where 
it is restricted to specific sites at the cell membrane. Again, this aspect has not been analyzed in protozoa.

As mentioned, in a small percentage of exponentially growing cells, an alternative mode of CVC biogenesis, designated above as "type II', is observed when a "young" CV pore forms at the lateral side of, but closely apposed to an old CVC (Klotz et al., 2003); old and new pore are both labeled with anti- $\gamma$-tubulin antibodies. Therefore, the assembly of $\gamma$-tubulin is the only known common denominator for an early step of CVC biogenesis.

Microtubules emanate from newly formed primordial CVCs. When they have reached their final length, microtubules may have to be stabilized, or they may reach their final length by ongoing destabilization. In metazoans, stabilization can be achieved, e.g. by attachment of additional proteins, such as +TIPs (Akhmanova \& Steinmetz, 2010; Mimori-Kiyosue \& Tsukita, 2003). Again, such proteins have not been identified in protozoa as yet. An alternative would be steady-state de-/repolymerization. In this case, Kif-type kinesins, known to regulate microtubule length in metazoan cells (Mayr et al., 2007; Niwa et al., 2012), could come into play, although they are also not yet known from protozoa. Remarkably, during microtubule growth, membranes of the smooth spongiome are tightly coupled to these microtubules of the radial arms, and calmodulin is considerably concentrated up to the pore (Fok et al., 2008). This may mutually stabilize microtubules and the attached membrane system.

Finally, it is known from Dictyostelium that myosin is a pore component serving for the attachment of the $\mathrm{CV}$ to the cell membrane (Jung et al., 2009). From all this, one can assign to cytoskeletal elements a key-function in the de novo biogenesis of CVCs.

\section{Calcium, phosphorylation processes and cell division}

$\mathrm{Ca}^{2+}$ is important for maintaining the integrity of the cell cortex. For instance, chelators dissociate alveolar sacs from the cell membrane (Stelly et al., 1991), and minor changes in local $\mathrm{Ca}^{2+}$ concentration can act as a morphogen. $\mathrm{Ca}^{2+}$ may be liberated by CRCs, type PtCRC-VI-2 and PtCRC-VI-3, which are located around the pore (Ladenburger \& Plattner, 2011). Although their localization is not known at the EM level, the number of sites where these molecules can be located is restricted. This can be either the CV moiety of the pore or any of the surrounding membranes. Association of such CRCs with the pore region is close enough to account for locally restricted $\mathrm{Ca}^{2+}$ signals. This is supported by the observation that PtCRCVI molecules are delivered by small vesicles to the cell cortex (Ladenburger \& Plattner, 2011). Altogether, this leaves us with the hypothesis that a $\mathrm{Ca}^{2+}$ signal may drive, or at least influence CVC biogenesis particularly of type I an assumption to be scrutinized in future work.

Cell cycle-dependent changes in the pattern of cortical protein phosphorylation accompany the restructuring of the cell surface in Paramecium (Sperling et al., 1991). Remarkably, mode I and II of de novo CVC biogenesis are reported from cells with high division activity (Allen et al., 1990; Klotz et al., 2003). Factors related to cell division activity may, therefore, be of paramount importance for de novo CVC biogenesis a hypothesis supported by a cursory observation of a NIMA-type protein kinase at the CV pore of Tetrahymena (Wloga et al., 2006); in mammalian neurons, it serves for the organization of the mitotic (Mayr et al., 2007) or cytoplasmic (Chang et al., 2009) microtubule apparatus. Though this is speculative at this time, further analysis along these lines may help to unravel some of the factors determining CVC biogenesis.

\section{Vesicle trafficking proteins around the pore and gene silencing experiments}

The SNAREs found at the pore are not restricted to this part of the organelle. However, their presence indicates the importance either of vesicle delivery to the pore region or their involvement in periodic opening of the pore by membrane fusion. Occurrence of some more SNARE types in all other parts of the CVC, except the decorated spongiome, suggests vesicle delivery over the entire organelle, and the same SNAREs have been found in nascent CVCs (Kissmehl et al., 2007; Schilde et al., 2006, 2010).

Posttranscriptional silencing of different organelle-resident SNAREs or of NSF affects the activity of the CVC, such as contraction periodicity and sensitivity to increased extracellular $\mathrm{Ca}^{2+}$ (Schönemann et al., 2013). CVC biogenesis is also disturbed by the silencing of several subunits of the $\mathrm{H}^{+}$-ATPase (Wassmer et al., 2005) and, interestingly, by silencing of an actin isoform, PtAct9 (Sehring et al., 2007). In some of these experiments, newly formed CVCs were mislocated specifically in cells of considerably deformed shape. Similar effects have been observed after PtSyx6 silencing (Schönemann et al., 2013). However, these effects are possibly an indirect consequence of silencing because of the following reasons. First, $\mathrm{H}^{+}$ATPase subunits are parts of the decorated spongiome and, thus, participate in biogenesis only at a late step. Second, no actin has been detected so far at the CVC (although its occurrence cannot strictly be excluded; see above). In these cases, the basic effect causing mislocation may, thus, be a disturbance in the overall cell morphology.

\section{Hypothesis for the chicken and egg problem of de novo biogenesis}

Specific proteins are localized to the CV pore, the site where biogenesis begins. However, are they located at this site to organize the new CVC or as a result of ongoing CVC organization? This takes us 50 years back when the cell surface pattern was recognized as an essential epigenetic factor for the formation and positioning of cortical elements in ciliates (Frankel, 2000) and for the CVC in particular (Nanney, 1966). However, we now can envisage specific aspects for in-depth scrutiny. De novo CVC formation is particularly striking during increased cell division activity, thus suggesting that the same factors are involved in CVC biogenesis and cell division. A comparison of centrioles and $\mathrm{CVC}$ organization sites suggests a role for $\gamma$-tubulin, centrin, calmodulin and NIMA-type kinase. As mentioned, installation of a new CVC at the cell surface requires the local exclusion of alveolar sacs to accommodate the pore a process whose regulation is unknown. Local $\mathrm{Ca}^{2+}$ signals, generated by 
specific $\mathrm{Ca}^{2+}$-channels (type $\mathrm{InsP}_{3} \mathrm{Rs} / \mathrm{RyRs}$ as occurring selectively in the pore region), may be important to establish a nucleation site for a primordial CVC. Expansion of the membranes of the newly forming organelle requires tubulin polymerization (and possibly posttranslational modification), SNAREs and any other proteins mandatory for vesicle delivery, as well as molecular motors and structural molecules involved in tubulation. Evidently, a variety of signals have to cooperate in an intriguing way for the de novo formation of a CVC. Many of the details, though currently hypothetical, are now amenable to experimental scrutiny.

\section{Acknowledgements}

I thank the respective coworkers for their continuous efforts as well as some of my colleagues for critically reading the text.

\section{Declaration of interest}

The author reports no conflicts of interest. The author alone is responsible for the content and writing of this article.

The author's work cited herein has been supported by grants from the German Research Council.

\section{References}

Akhmanova A, Steinmetz MO. (2010). Microtubule +TIPs at a glance. J Cell Sci 123:3415 9.

Allen RD. (2000). The contractile vacuole and its membrane dynamics. Bioessays 22:1035 42

Allen RD, Naitoh Y. (2002). Osmoregulation and contractile vacuoles in protozoa. Int Rev Cytol 215:351 94.

Allen RD, Ueno MS, Pollard LW, Fok AK. (1990). Monoclonal antibody study of the decorated spongiome of contractile vacuole complexes of Paramecium. J Cell Sci 96:469 75.

Andersen OS, Koeppe RE. (2007). Bilayer thickness and membrane protein function: an energetic perspective. Annu Rev Biophys Biomol Struct 36:107 30 .

Blumenthal R. (1987). Membrane fusion. Curr Top Membr Transp 29: 20354.

Bright LJ, Kambesis N, Nelson SB, et al. (2010). Comprehensive analysis reveals dynamic and evolutionary plasticity of Rab GTPases and membrane traffic in Tetrahymena thermophila. PLoS Genet 6: e1001155.

Chang J, Baloh RH, Milbrandt J. (2009). The NIMA family kinase Nek3 regulates microtubule acetylation in neurons. J Cell Sci 122:2274 82 .

Coste B, Mathur J, Schmidt M, et al. (2010). Piezol and Piezo2 are essential components of distinct mechanically activated cation channels. Science 330:55 60.

Desai A, Mitchison TJ. (1997). Microtubule polymerization dynamics. Annu Rev Cell Dev Biol 13:83 117.

Dobbelaere J, Josué F, Suijkerbuijk S, et al. (2008). A genome wide RNAi screen to dissect centriole duplication and centrosome matur ation in Drosophila. PloS Biol 6:e224.

Elde NC, Morgan G, Winey M, et al. (2005). Elucidation of clathrin mediated endocytosis in Tetrahymena reveals an evolutionarily convergent recruitment of dynamin. PloS Genet 1:e52.

Essid M, Gopaldass N, Yoshida K, et al. (2012). Rab8a regulates the exocyst mediated kiss and run discharge of the Dictyostelium contractile vacuole. Mol Biol Cell 23:1267 82.

Fok AK, Clarke M, Ma L, Allen RD. (1993). Vacuolar $\mathrm{H}^{+}$ATPase of Dictyostelium discoideum. A monoclonal antibody study. J Cell Sci 106:1103 13 .

Fok AK, Aihara MS, Ishida M, Allen RD. (2008). Calmodulin localization and its effects on endocytic and phagocytic membrane trafficking in Paramecium multimicronucleatum. J Eukaryot Microbiol 55:481 91.
Frankel J. (2000). Cell biology of Tetrahymena thermophila. Meth Cell Biol 62:27 125.

Fujiu K, Nakayama Y, Iida H, et al. (2011). Mechanoreception in motile flagella of Chlamydomonas. Nature Cell Biol 13:630 3.

Gaertig J, Cruz MA, Bowen J, et al. (1995). Acetylation of lysine 40 in alpha tubulin is not essential in Tetrahymena thermophila. J Cell Biol 129:1301 10.

Gerisch G, Heuser J, Clarke M. (2002). Tubular vesicular transformation in the contractile vacuole system of Dictyostelium. Cell Biol Int 26: 84552.

Gogendeau D, Klotz C, Arnaiz O, et al. (2008). Functional diversifica tion of centrins and cell morphological complexity. J Cell Sci 121: 6574.

Griffiths GM, Tsun A, Stinchcombe JC. (2010). The immunological synapse: a focal point for endocytosis and exocytosis. J Cell Biol 189: 399406.

Grønlien HK, Stock C, Aihara MS, et al. (2002). Relationship between the membrane potential of the contractile vacuole complex and its osmoregulatory activity in Paramecium multimicronucleatum. J Exp Biol 205:3261 70 .

Harris TJC, Peifer M. (2005). The positioning and segregation of apical cues during epithelial polarity establishment in Drosophila. J Cell Biol 170:813 23.

Hauser K, Pavlovic N, Klauke N, et al. (2000). Green fluorescent protein tagged sarco(endo)plasmic reticulum $\mathrm{Ca}^{2+}$ ATPase overex pression in Paramecium cells: isoforms, subcellular localization, biogenesis of cortical calcium stores and functional aspects. Mol Microbiol 37:773 87.

Heath RJ, Insall RH. (2008). Dictyostelium MEGAPs: F BAR domain proteins that regulate motility and membrane tubulation in contractile vacuoles. J Cell Sci 121:1054 64.

Heider MR, Munson M. (2012). Exorcising the exocyst complex. Traffic 13:898 907 .

Henrique D, Schweisguth F. (2003). Cell polarity: the ups and downs of the Par6/aPKC complex. Curr Op Genet Dev 13:341 50.

Heuser J. (2006). Evidence for recycling of contractile vacuole membrane during osmoregulation in Dictyostelium amoebae a tribute to Günther Gerisch. Eur J Cell Biol 85:859 71 .

Heuser J, Zhu Q, Clarke M. (1993). Proton pumps populate the contractile vacuoles of Dictyostelium amoebae. J Cell Biol 121: 131127.

Hodges ME, Scheumann N, Wickstead B, et al. (2010). Reconstructing the evolutionary history of the centriole from protein components. J Cell Sci 123:1407 13.

Huang M, Gu G, Ferguson EL, Chalfie M. (1995). A stomatin like protein necessary for mechanosensation in C. elegans. Nature 378: 2925.

Iftode F, Cohen J, Ruiz F, et al. (1989). Development of surface pattern during division in Paramecium. I. Mapping of duplication and reorganization of cortical cytoskeletal structures in the wild type. Development 105:191 211.

Ingham PW, Nakano Y, Seger C. (2011). Mechanisms and functions of Hedgehog signalling across the metazoa. Nature Rev Genet 12: 393406.

Jahn R, Scheller RH. (2006). SNAREs engines for membrane fusion. Nat Rev Mol Cell Biol 7:631 43.

Jerka Dziadosz M, Jenkins LM, Nelsen EM, et al. (1995). Cellular polarity in ciliates: persistence of global polarity in a disorganized mutant of Tetrahymena thermophila that disrupts cytoskeletal organ ization. Dev Biol 169:644 61.

Joberty G, Petersen C, Gao L, Macara IG. (2000). The cell polarity protein Par6 links Par3 and atypical protein kinase C to Cdc42. Nat Cell Biol 2:531 9.

Jung G, Titus MA, Hammer JA. (2009). The Dictyostelium type V myosin MyoJ is responsible for the cortical association and motility of contractile vacuole membranes. J Cell Biol 186:555 70.

Kalebic N, Martinez C, Perla, E, et al. (2013). Tubulin acetyltransferse aTAT1 destabilizes microtubules independently of its acetylation activity. Mol Cell Biol 33:1114 23.

Kissmehl R, Froissard M, Plattner H, et al. (2002). NSF regulates membrane traffic along multiple pathways in Paramecium. J Cell Sci 115:3935 46.

Kissmehl R, Schilde C, Wassmer T, et al. (2007). Molecular identifi cation of 26 syntaxin genes and their assignment to the different trafficking pathways in Paramecium. Traffic 8:523 42. 
Klotz C, Ruiz F, Garreau de Loubresse N, et al. (2003). Gamma tubulin and MTOCs in Paramecium. Protist 154:193 209.

Komsic Buchmann K, Stephan LM, Becker B. (2012). The SEC6 protein is required for contractile vacuole function in Chlamydomonas reinhardtii. J Cell Sci 125:2885 95.

Kung C, Martinac B, Sukharev S. (2010). Mechanosensitive channels in microbes. Annu Rev Microbiol 64:313 29.

Ladenburger EM, Plattner H. (2011). Calcium release channels in Paramecium. Genomic expansion, differential positioning and partial transcriptional elimination. PLoS One 6:e27111.

Ladenburger EM, Korn I, Kasielke N, et al. (2006). An Ins(1,4,5) $\mathrm{P}_{3}$ receptor in Paramecium is associated with the osmoregulatory system. J Cell Sci 119:3705 17.

Lapatsina L, Brand J, Poole K, et al. (2012). Stomatin domain proteins. Eur J Cell Biol 91:240 5.

Marchesini N, Ruiz FA, Vieira M, Docampo R. (2002). Acidocalcisomes are functionally linked to the contractile vacuole of Dictyostelium discoidium. J Biol Chem 277:8146 53.

Mayr MI, Hummer S, Bormann J, et al. (2007). The human kinesin Kif18A is a motile microtubule depolymerase essential for chromo some congression. Curr Biol 17:48898.

Mim C, Unger VM. (2012). Membrane curvature and its generation by BAR proteins. Trends Biochem Sci 37:526 33 .

Mimori Kiyosue Y, Tsukita S. (2003). "Search and capture" of micro tubules through plus end binding proteins (+TIPs). J Biochem 134: 3216.

Momayezi M, Kersken H, Gras U, et al. (1986). Calmodulin in Paramecium tetraurelia: localization from the in vivo to the ultrastructural level. J Histochem Cytochem 34:1621 38.

Montalvetti A, Rohloff P, Docampo R. (2004). A functional aquaporin co localizes with the vacuolar proton pyrophosphatase to acidocalci somes and the contractile vacuole complex of Trypanosoma cruzi. J Biol Chem 279:38673 82.

Nanney DL. (1966). Cortical integration in Tetrahymena: an exercise in cytogeometry. J Exp Zool 161:307 17

Nishihara E, Yokota E, Tazaki A, et al. (2008). Presence of aquaporin and V ATPase on the contractile vacuole of Amoeba proteus. Biol Cell 100:179 88 .

Niwa S, Nakajima K, Miki H, et al. (2012). KIF19A is a microtubule depolymerizing kinesin for ciliary length control. Dev Cell 23: 116775 .

Nolta KV, Padh H, Steck TL. (1993). An immunocytochemical analysis of the vacuolar proton pump in Dictyostelium discoideum. J Cell Sci 105:849 59 .

Numata O, Gonda K. (2001). Determination of division plane and organization of contractile ring in Tetrahymena. Cell Struct Funct 26: 593601

Ohki S. (1988). Surface tension, hydration energy and membrane fusion. In: Ohki S, Doyle D, Flanagan TD, et al., eds. Molecular mechanisms of fusion. New York: Plenum Press, 12338.

Palmer KJ, Watson P, Stephens DJ. (2005). The role of microtubules in transport between the endoplasmic reticulum and Golgi apparatus in mammalian cells. Biochem Soc Symp 72:1 13 .

Pang ZP, Südhof TC. (2010). Cell biology of $\mathrm{Ca}^{2+}$ triggered exocytosis. Curr Opin Cell Biol 22:496 505.

Plattner H. (2010). Membrane trafficking in protozoa: SNARE proteins, $\mathrm{H}^{+}$ATPase, actin, and other key players in ciliates. Int Rev Cell Mol Biol 280:79 184

Plattner H, Westphal C, Tiggemann R. (1982). Cytoskeleton secretory vesicle interactions during the docking of secretory vesicles at the cell membrane in Paramecium tetraurelia cells. J Cell Biol 92:368 77.

Plattner H, Sehring IM, Mohamed IK, et al. (2012). Calcium signaling in closely related protozoan groups (Alveolata): non parasitic ciliates (Paramecium, Tetrahymena) vs. parasitic Apicomplexa (Plasmodium, Toxoplasma). Cell Calcium 51:351 82.

Ren G, Vajjhala P, Lee JS, et al. (2006). The BAR domain proteins: molding membranes in fission, fusion, and phagy. Microbiol Mol Biol Rev 70:37 120.

Reuter AT, Stuermer CAO, Plattner H. (2013). Identification, localiza tion, and functional implications of the microdomain forming stomatin family in the ciliated protozoan Paramecium. Eukaryot Cell 12:529 44 .

Schilde C, Wassmer T, Mansfeld J, et al. (2006). A multigene family encoding R SNAREs in the ciliate Paramecium tetraurelia. Traffic 7: 44055 .
Schilde C, Lutter K, Kissmehl R, Plattner H. (2008). Molecular identification of a SNAP 25 like SNARE protein in Paramecium. Eukaryot Cell 7:1387 402.

Schilde C, Schönemann B, Sehring IM, Plattner H. (2010). Distinct subcellular localization of a group of synaptobrevin like SNAREs in Paramecium tetraurelia and effects of silencing of the SNARE specific chaperone NSF. Eukaryot Cell 9:288 305.

Schönemann B, Bledowski A, Sehring IM, Plattner H. (2013). A set of SNARE proteins in the contractile vacuole complex of Paramecium regulates cellular calcium tolerance and also contributes to organelle biogenesis. Cell Calcium 53:204 16 .

Sehring IM, Mansfeld J, Reiner C, et al. (2007). The actin multigene family of Paramecium tetraurelia. BMC Genomics 8:82.

Shang Y, Li B, Gorovsky MA. (2002). Tetrahymena thermophila contains a conventional $\gamma$ tubulin that is differentially required for the maintenance of different microtubule organizing centers. J Cell Biol 158:1195 206

Shen H, Pirruccello M, De Camilli P. (2012). SnapShot: membrane curvature sensors and generators. Cell 150:1300.e1 2.

Shilagardi K, Li S, Luo F, et al. (2013). Actin propelled invasive membrane protrusions promote fusogenic protein engagement during cell cell fusion. Science 340:359 63 .

Sperling L, Keryer G, Ruiz F, Beisson J. (1991). Cortical morphogenesis in Paramecium: a transcellular wave of protein phosphorylation involved in ciliary rootlet disassembly. Dev Biol 148:205 18.

Sriskanthadevan S, Lee T, Lin Z, et al. (2009). Cell adhesion molecule $D d$ CAD 1 is imported into contractile vacuoles by membrane invagination in a $\mathrm{Ca}^{2+}$ and conformation dependent manner. J Biol Chem 284:36377 86

Stelly N, Mauger JP, Claret M, Adoutte A. (1991). Cortical alveoli of Paramecium: a vast submembranous calcium storage compartment. J Cell Biol 113:103 12.

Stemm Wolf AJ, Morgan G, Giddings TH, et al. (2005). Basal body duplication and maintenance require one member of the Tetrahymena thermophila centrin gene family. Mol Biol Cell 16:3606 19.

Stenmark H. (2009). Rab GTPases as coordinators of vesicle traffic. Nat Rev Mol Cell Biol 10:513 25.

Stock C, Grønlien HK, Allen RD. (2002). The ionic composition of the contractile vacuole fluid of Paramecium mirrors ion transport across the plasma membrane. Eur J Cell Biol 81:505 15.

Sugino K, Tominaga T, Allen RD, Naitoh Y. (2005). Electrical properties and fusion dynamics of in vitro membrane vesicles derived from separate parts of the contractile vacuole complex of Paramecium multimicronucleatum. J Exp Biol 208:3957 69.

Tani T, Allen RD, Naitoh Y. (2001). Cellular membranes that undergo cyclic changes in tension: direct measurement of force generation by an in vitro contractile vacuole of Paramecium multimicronucleatum. J Cell Sci 114:785 95.

Tominaga T, Allen R, Naitoh Y. (1998a). Electrophysiology of the in situ contractile vacuole complex of Paramecium reveals its membrane dynamics and electrogenic site during osmoregulatory activity. J Exp Biol 201:451 60 .

Tominaga T, Allen R, Naitoh Y. (1998b). Cyclic changes in the tension of the contractile vacuole complex membrane control its exocytotic cycle. J Exp Biol 201:2647 58.

Wassmer T, Froissard M, Plattner H, et al. (2005). The vacuolar proton ATPase plays a major role in several membrane bounded organelles in Paramecium. J Cell Sci 118:2813 25.

Wassmer T, Kissmehl R, Cohen J, Plattner H. (2006). Seventeen a subunit isoforms of Paramecium V ATPase provide high special ization in localization and function. Mol Biol Cell 17:917 30.

Wassmer T, Sehring IM, Kissmehl R, Plattner H. (2009). The V ATPase in Paramecium: functional specialization by multiple gene isoforms. Eur J Physiol 457:599 607.

Wloga D, Camba A, Rogowski K, et al. (2006). Members of the NIMA related kinase family promote disassembly of cilia by multiple mechanisms. Mol Biol Cell 17:2799 810.

Yano J, Rajendran A, Valentine MS, et al. (2013). Proteomic analysis of the cilia membrane of Paramecium tetraurelia. J Proteom 78 11322

Yoon Y, Pitts KR, Dahan S, McNiven MA. (1998). A novel dynamin like protein associates with cytoplasmic vesicles and tubules of the endoplasmic reticulum in mammalian cells. J Cell Biol 140:779 93. 